\title{
Single-shot wavefront characterization of high topological charge extreme-ultraviolet vortex
}

\begin{abstract}
A. K. Pandey, O. Guilbaud, F. Sanson, I. Papagiannouli, A. Kabacinski, et al.
\end{abstract}

A. K. Pandey, O. Guilbaud, F. Sanson, I. Papagiannouli, A. Kabacinski, F. Tissandier, F. Harms, G. Beaugrand, G. Dovillaire, E. Baynard, J. Demailly, B. Lucas, O. Neveu, M. Pittman, D. Ros, S. Sebban, Ph. Balcou, S. Kazamias, "Single-shot wavefront characterization of high topological charge extremeultraviolet vortex," Proc. SPIE 11886, International Conference on X-Ray Lasers 2020, 118860L (8 July 2021); doi: 10.1117/12.2592326 


\title{
Single-shot wavefront characterization of high topological charge extreme-ultraviolet vortex
}

\author{
A. K. Pandey ${ }^{* a}$, O. Guilbaud ${ }^{\text {a }}$, F. Sanson ${ }^{\text {a,b }}$, I. Papagiannouli a , A. Kabacinski ${ }^{c}$, F. Tissandier ${ }^{c}$, F. \\ Harms $^{\mathrm{d}}$, G. Beaugrand ${ }^{\mathrm{d}}$, G. Dovillaire ${ }^{\mathrm{d}}$, E. Baynard ${ }^{\mathrm{a}}$, J. Demailly ${ }^{\mathrm{a}}$, B. Lucas ${ }^{\mathrm{a}}$, O. Neveu ${ }^{\mathrm{a}}$, M. \\ Pittman $^{\text {a }}$, D. Ros ${ }^{\text {a }}$, S. Sebban ${ }^{\text {c }}, \mathrm{Ph}$. Balcou $^{\mathrm{e}}, \mathrm{S}$. Kazamias ${ }^{\mathrm{a}}$ \\ ${ }^{a}$ Laboratoire Irène Joliot-Curie, Université Paris-Saclay, UMR CNRS, Rue Ampère, Bâtiment \\ 200,F-91898, Orsay Cedex, France; ${ }^{b}$ Amplitude Laser Group, Scientific Business Unit-Lisses \\ Operations, 2/4 rue du Bois Chaland, 91090 Lisses, France; ${ }^{\mathrm{c}}$ Laboratoire d'Optique Appliquée, \\ ENSTA Paris, Ecole Polytechnique, CNRS, Institut Polytechnique de Paris, 828 Boulevard des \\ Maréchaux, F-91761 Palaiseau cedex, France; d Imagine Optic, 18, rue Charles de Gaulle, 91400 \\ ORSAY, France; ${ }^{\mathrm{e}}$ Univ. Bordeaux-CNRS-CEA, CELIA, UMR 5107, Talence, France.
}

\begin{abstract}
We present an experimental intensity and wavefront characterization of the infrared vortex driver as well as the extreme ultraviolet vortex obtained through high harmonic generation in an extended generation medium. In a loose focusing geometry, an intense vortex beam obtained through phase-matched absorption-limited high harmonic generation in a 15 $\mathrm{mm}$ long Argon filled gas-cell permits single-shot characterization of the vortex structure. Moreover, our study validates the multiplicative law of momentum conservation even for such an extended generation medium.
\end{abstract}

Keywords: EUV vortex, high-harmonic generation, photon orbital angular momentum, EUV wavefront metrology

\section{INTRODUCTION}

It is known that light beams carry linear as well as angular momenta. The spin angular momentum (SAM) of photons is related to polarization [1]. On the other hand, orbital angular momentum (OAM) is independent of the polarization and is related to the wavefront instead [2]. Such beams, also known as optical vortices, exhibit a transverse helical phase with $e^{i l \Phi}$ dependence around the beam propagation axis. A rapid phase rotation around the center results in an on-axis phase singularity yielding a donut-like intensity profile with a dark-hole at the center. There are two main attributes for such beams: (1) the topological charge $l$ is the number of $2 \pi$ phase shifts along the azimuthal coordinate $\Phi$ of the beam, and (2) for a vortex beam of topological charge $l$, each photon carries an OAM of $l \hbar$ [2]. Moreover, unlike the intrinsic nature of SAM, the OAM of helically phased beams can either be intrinsic or extrinsic [3].

Owing to the numerous applications of optical vortices, the generation, and characterization of light beams carrying topological singularities has received increasing attention in the recent past [4]. Besides, high harmonic generation (HHG) in rare gases has proven to be a handy way to generate extreme-ultraviolet (EUV) vortices of low and high topological charges [5-7]. HHG, when driven by a driver of topological charge $l_{1}$, follows the perturbative energy conversion law $l_{q}=$ $q l_{1}$, where $l_{q}$ is the topological charge of $q^{\text {th }}$ harmonic [5, 7]. In this work, we present experimental intensity and wavefront characterization of very high-charge EUV vortex obtained through high harmonic generation in an Argon filled gas-cell of $10 \mathrm{~mm}$ and $15 \mathrm{~mm}$ lengths. In both cases, we validate the multiplicative law $l_{q}=q l_{1}$ for the $25^{\text {th }}$ harmonic of the driving beam, hence ruling out any propagation effects [8] even in a $15 \mathrm{~mm}$ long generation medium. Moreover, efficient HHG in the longer generation medium allowed intensity and wavefront characterization in single-shot mode. The ability to perform such a characterization of the high-charge vortex structure provides a unique opportunity to investigate the effect of phase-matching and other experimental parameters on the modal content of EUV vortex beam in single-shot mode, which will be reported in the forthcoming study.

*alok-kumar.pandey@u-psud.fr

International Conference on X-Ray Lasers 2020, edited by Davide Bleiner,

Proc. of SPIE Vol. 11886, 118860L · C 2021 SPIE · CCC code:

0277-786X/21/\$21 - doi: $10.1117 / 12.2592326$ 


\section{EXPERIMENTAL SETUP AND RESULTS.}

The schematic of the experimental setup is presented in Fig. 1. High-harmonic generation is driven by the low-energy beamline of the LASERIX multibeam platform at the University of Paris-Saclay [9]. The near-infrared (IR) beam of central wavelength $815 \mathrm{~nm}$, pulse duration $\sim 45 \mathrm{fs}$, and diameter $\sim 24 \mathrm{~mm}$ at $1 / e^{2}$, drives HHG at a $10 \mathrm{~Hz}$ repetition rate. The energy of the IR beam can be continuously varied using a half-waveplate and polarizer. An iris of variable diameter placed before the focusing optics is used to optimize the phase matching. Moreover, the experimental setup is adapted to readily switch between different focusing conditions and gas-cell lengths. The focusing lens, as well as gas-cell, are mounted on motorized translation stages, allowing precise displacement of the generation medium with respect to the beam waist.

The HHG beamline is equipped with wavefront sensors for near-IR $(800 \pm 50 \mathrm{~nm})$ and EUV to soft X-ray (40 to $4 \mathrm{~nm}$ ) spectral range. The diagnostic to the amplitude and phase of the IR driver is provided by a Shack-Hartmann wavefront sensor (HASO4 FIRST, Imagine Optic). Given sensor consists of an array of microlenses $(32 \times 40)$ providing RMS tilt measurement sensitivity of $\sim 5 \mu \mathrm{rad}$ and an absolute wavefront measurement accuracy of $\sim \lambda / 100$ at the calibrated wavelength. The amplitude and phase of the upconverted high-harmonic beam are characterized using an Extreme ultraviolet Hartmann sensor (EUV HASO, Imagine Optic) located $\sim 1.98 \mathrm{~m}$ following the generation medium. The EUV HASO comprises a Hartmann mask of $72 \times 72$ square micro-hole array placed before a back-illuminated EUV CCD (Pixis XO 1024B; pixel size $13 \mu \mathrm{m}$ ). Each of these subpupils samples a part of the incident wavefront to yield local intensity and wavevector direction with a tilt measurement sensitivity of $\sim 0.05 \mu \mathrm{rad} \mathrm{Rms}$. This leads to an absolute wavefront measurement accuracy of $\sim \lambda / 50$ at $30 \mathrm{~nm}$. In contrast to diffractive [10] or interferometric techniques [5], using a wavefront sensor yields a complete amplitude and phase of the vortex beam. It is also to note that wavefront measurements of optical vortices have previously been used to measure the Poynting vector skew angle [11], and most recently, revealed the multimodal nature of EUV vortices obtained through HHG in a noble gas [12].

The experimental setup is also equipped with a high-resolution, monochromatic, EUV imaging system that allows us to characterize the near-field intensity profile of the high-harmonic source. Furthermore, the given system can be used to image the exit of gas-cell onto two different EUV CCDs with a magnification factor of $\sim 6.5$ and $\sim 11$, leading to a spatial resolution of $2 \mu \mathrm{m}$ and $\sim 1.2 \mu \mathrm{m}$ at the object plane, respectively.

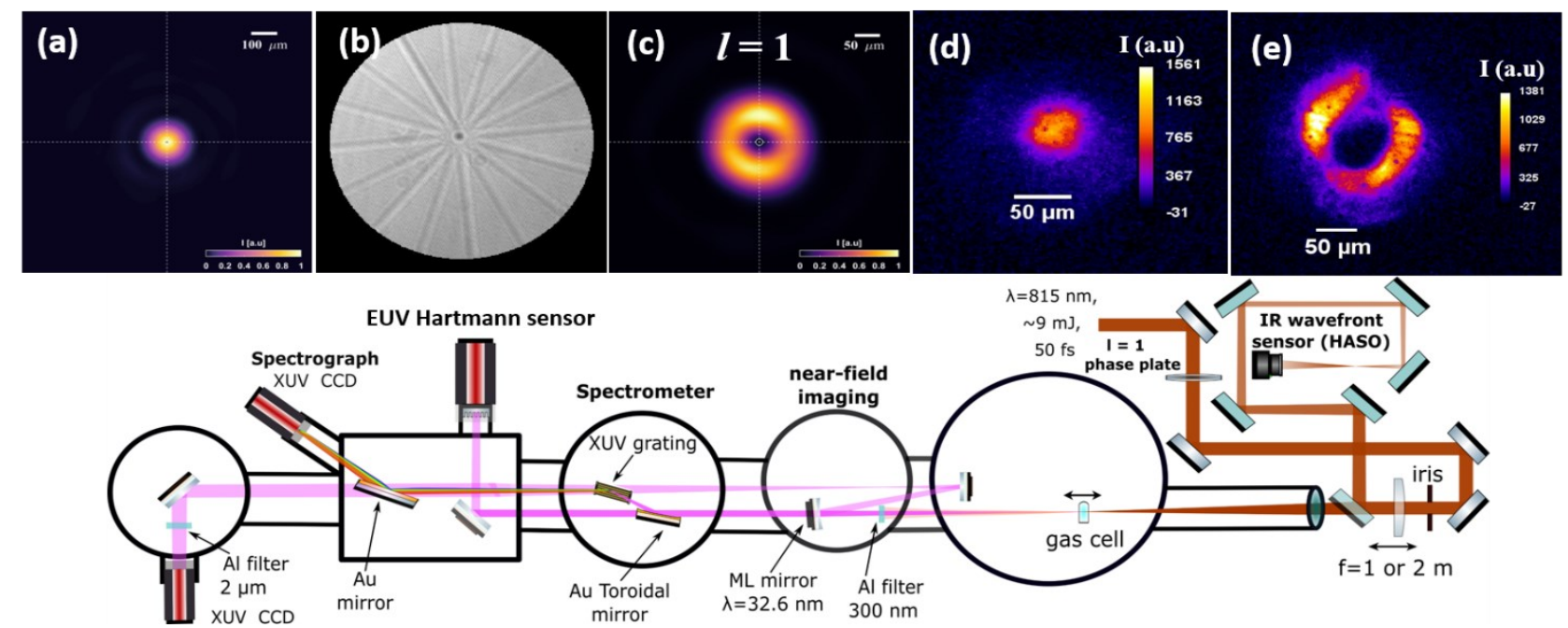

Fig. 1. Schematic of the experimental setup. A high damage threshold segmented spiral phase plate of a unit topological charge is inserted in the incoming beam. The resulting IR vortex driver is loosely focused by a $1 \mathrm{~m}$ or $2 \mathrm{~m}$ focal length lens into $10 \mathrm{~mm}$ or $15 \mathrm{~mm}$ Argon filled gas-cell, respectively. A diaphragm before the focusing optics is used to optimize the phase matching. The motorized translation stage of the focusing lens allows adjustment of the IR beam waist with respect to the generation medium. Intensity and phase characterization to the driver is provided by a Shack-Hartmann wavefront sensor, whereas a EUV Hartmann sensor ( $\lambda=4$ to $40 \mathrm{~nm})$ is used to fully characterize the upconverted $25^{\text {th }}$ harmonic of the IR driver. A high magnification monochromatic EUV (32.6 $\left.\mathrm{nm}\right)$ imaging system composed of a near-normal incidence concave mirror (focal length $500 \mathrm{~mm}$ ) and flat mirrors for beam steering, facilitates nearfield intensity characterization of the HHG source. Inset figure (a) shows the intensity profile of the IR Gaussian beam at the waist. The image of the segmented phase plate of unit topological charge is shown in (b), whereas (c) depicts the resulting doughnut-like intensity profile of the IR vortex at the waist. Nearfield intensity profile of the HHG source for gaussian (d) and vortex (e) IR driver is acquired using EUV imaging system with a magnification factor of $\sim 6.5$. 
The IR-vortex beam is generated by inserting a 16-segment spiral phaseplate [13] designed for wavelength $815 \mathrm{~nm}$ (HoloOr) into the incoming Gaussian beam. The phaseplate bears antireflective coating on both the faces and the resulting transmission efficiency is $\sim 99.9 \%$. To characterize the infrared vortex, the incoming beam is sampled and guided to the Shack-Hartmann wavefront sensor. The sensor is placed $\sim 300 \mathrm{~mm}$ after the waist. A typical raw-Hartmanngram is shown in Fig. 3 (a). The measured slopes, which is the local phase-front tilt sampled by each micro-lens of the wavefront sensor is shown in figure 3(b). The slopes exhibit a clockwise rotation around the central phase singularity. From the raw Hartmanngram, the intensity and wavefront of the vortex beam are reconstructed. The corresponding wavefronts are depicted in Fig. 3 (c, d). A clear phase variation of $\sim 1 \lambda$ signifies a unit topological charge, whereas a clockwise and an anticlockwise rotation of the wavefront is related to the positive and negative signs of the charge. Moreover, utilizing the Fourier Transform relationship between the topological charge $l$ and azimuthal angle $\Phi$, the OAM spectrum can be retrieved without the need for an analytical basis [14]. Thanks to the Shack-Hartmann wavefront sensor, such an analysis using the experimentally characterized amplitude and phase of IR-vortex indicates $\sim 91 \%$ contribution to $l_{1}= \pm 1$ azimuthal mode.

To generate high harmonics, the IR-vortex beam is loosely focused in the Argon-filled gas-cell. After removing the residual infrared beam with a metallic $\left(300 \mathrm{~nm}\right.$; Al) filter, the HHG beam is guided to the EUV wavefront sensor through a $45^{\circ}$ multilayer dielectric mirror acting as a spectral filter. The $25^{\text {th }}$ harmonic centered at the wavelength of $32.6 \mathrm{~nm}$ is spectrally selected and guided to the wavefront sensor. The spectral tuning of the harmonic beam is realized through IR spectral phase control using an acousto-optic modulator (Dazzler, FASTLITE). The extinction of the adjacent harmonic orders is over $90 \%$ [15]. The usage of such a mirror facilitates wavefront analysis of only the selected harmonic order, hence making the data interpretation unambiguous.
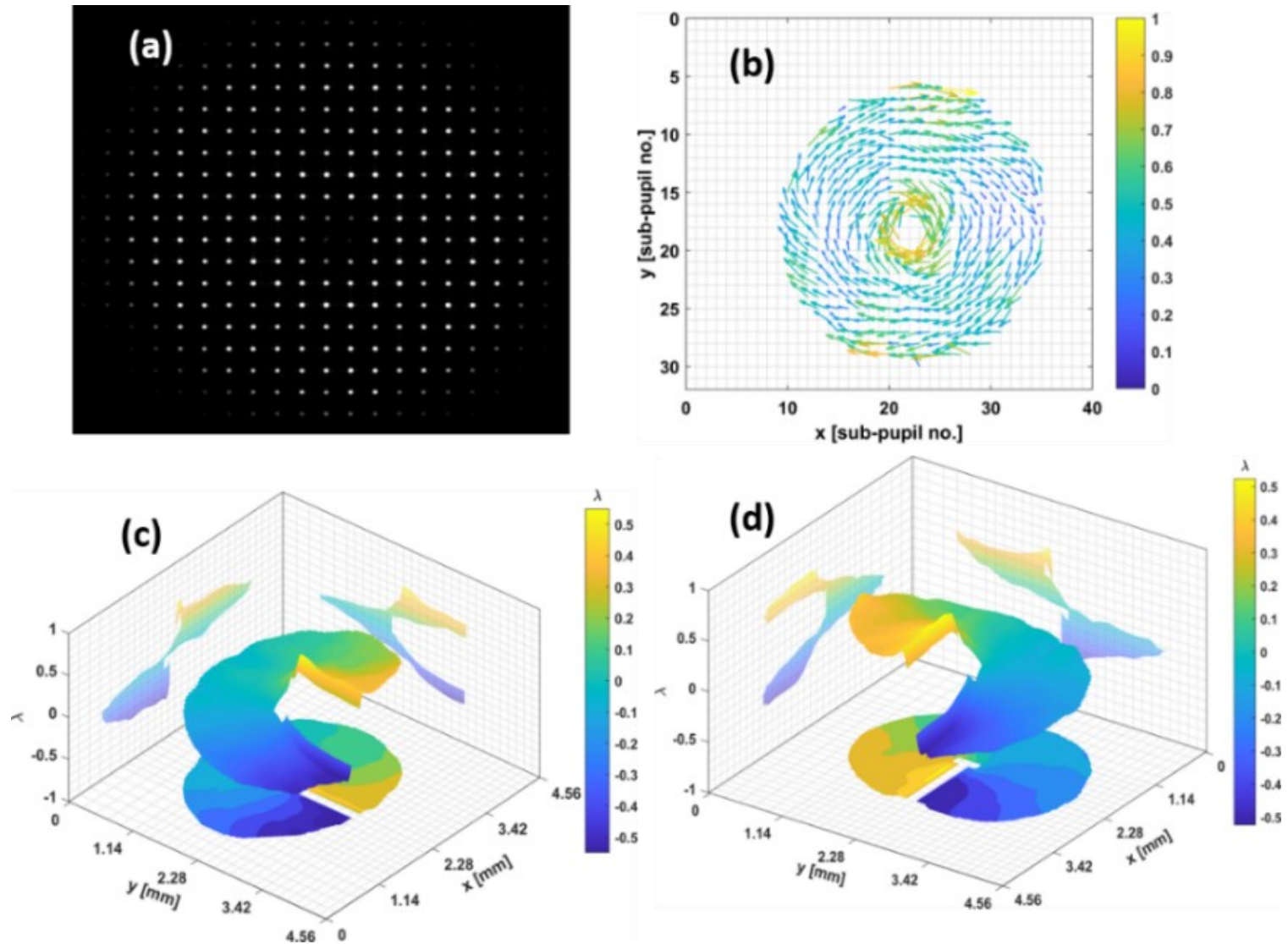

Fig. 2. Characterization of the infrared vortex: (a) an example raw Hartmanngram. (b) Quiver plot of the measured slopes indicating a clockwise rotation around the central phase singularity. (c, d) Three-dimensional representation of wavefront in the unit of $\lambda$.

For the $10 \mathrm{~mm}$ long gas-cell, the IR-vortex beam is apertured with an iris of diameter $\sim 13 \mathrm{~mm}$ and is focused using a plano-convex lens of focal length $1 \mathrm{~m}$. In the given focusing condition, an optimum HHG-OAM signal level is observed 
for a laser energy of $\sim 4.5 \mathrm{~mJ}$ after the diaphragm and 24 mbar gas-pressure. The gas-cell is positioned to have the waist of the driving beam in the center. The reconstructed intensity and wavefront of the 25 th harmonic for $l_{1}= \pm 1$ is shown in Fig. 3. Efficient HHG in $10 \mathrm{~mm}$ long gas-cell allows acquiring Hartmanngrams with high signal-to-noise by accumulating just 5 laser-shots. A continuous and smooth phase variation can be seen in Fig. 3(b) and 3(d). Moreover, the peak-to-valley phase variation is $\sim 25 \lambda$ in both cases validating the linear conversion law $l_{q}=q l_{1}$. This implies that even in a long generation medium, the EUV vortex retains the high-charge helical wavefront. Numerically backpropagated intensity profile utilizing the amplitude and phase of EUV vortex generated using $l_{1}=-1$ beam is depicted in Fig. 3 (e). A good agreement between the numerical and experimental nearfield intensity profile showed in Fig. 3 (f) indicates the highfidelity of Hartmann sensor data.
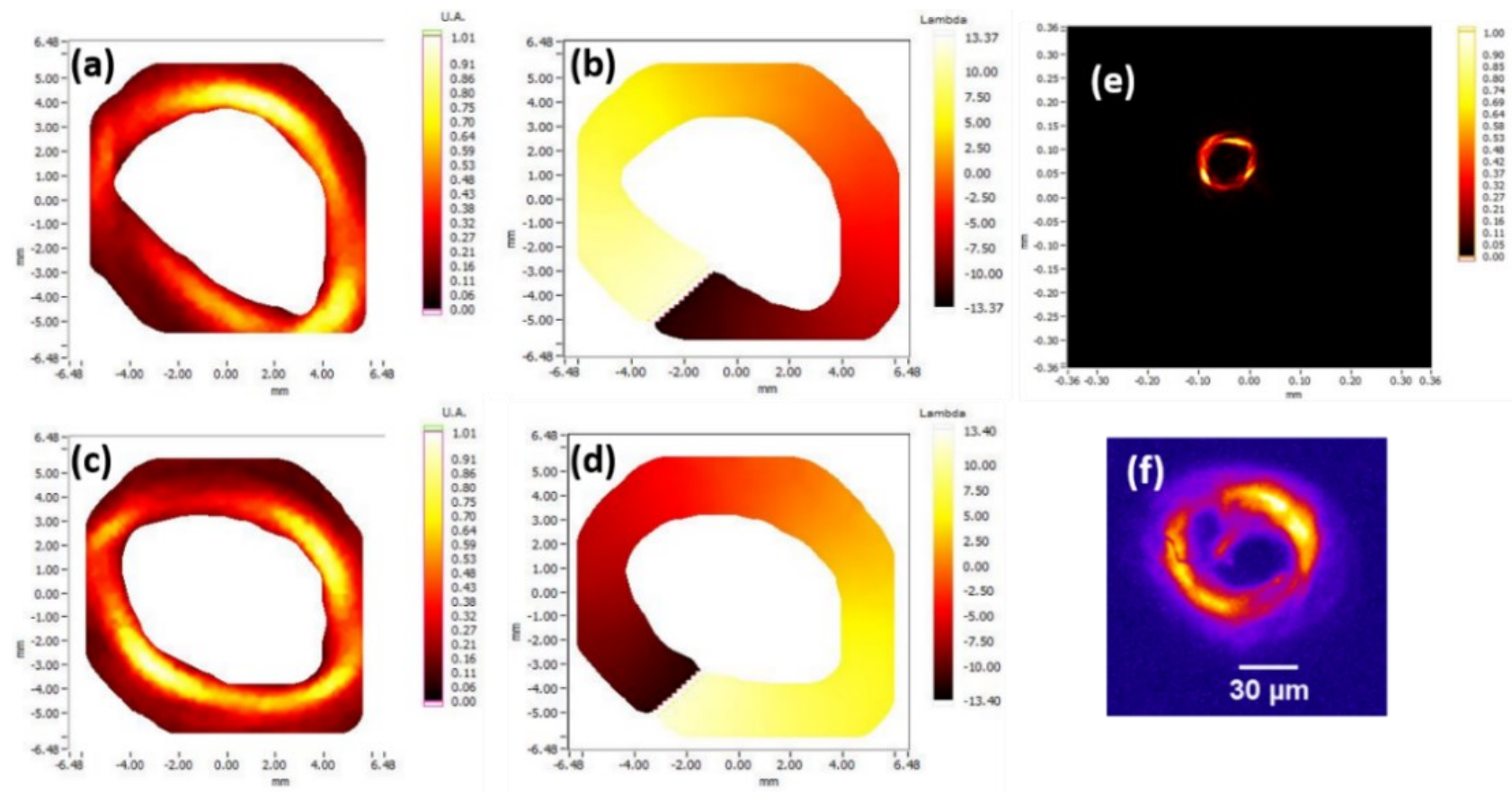

Fig. 3. Characterization of EUV vortex generated in $10 \mathrm{~mm}$ long Argon filled gas-cell. Reconstructed intensity (a) and wavefront (b) of EUV vortex generated using $l_{1}=-1$ infrared beam. Numerically retro-propagated intensity profile to the waist is shown in (c) whereas, an experimental near-field intensity profile acquired using the EUV imaging system (magnification $\sim 6.5$ ) is presented in (f). In (c, d) intensity and wavefront of EUV vortex for $l_{1}=+1$ is shown. The number of laser-shots accumulated is 5 for these cases.

In the next step, to generate the EUV vortex in a further long medium, we switch to 2-meter focusing geometry. The incoming vortex beam of topological charge $l_{1}=-1$ is clipped using an iris of diameter $\sim 20 \mathrm{~mm}$ and focused into $15 \mathrm{~mm}$ long Argon filled gas-cell. The Rayleigh length $z_{r}$ is $\sim 8 \mathrm{~mm}$ under these conditions. The confocal parameter $2 z_{r}$ still exceeds the length of the gas-cell, hence facilitating the generation of intense EUV vortices while satisfying the phasematching conditions. The driving beam has $\sim 11 \mathrm{~mJ}$ after the iris. The intensity profile of the IR-vortex beam at the waist in the given condition is shown in Fig. 1 (c). In contrast to the prior focusing condition, an optimum signal level was observed for $\sim 12$ mbar gas-pressure. Besides, an efficient HHG in a further long generation medium allowed single-shot amplitude and phase characterization of the EUV vortex beam. A single-shot raw Hartmanngram of the upconverted vortex is shown in Fig. 4 (a). A quiver plot of the measured slopes is shown in Fig. 4 (b). Similar to the rotating slopes of the IR driver presented in Fig. 2 (b), the EUV vortex also exhibit spiraling slopes around the central phase singularity. From the raw Hartmanngram, intensity and wavefront are reconstructed. The resulting wavefront is shown in Fig. 4(d) bears a smooth and continuous wavefront rotation. Moreover, the peak-to-valley wavefront variation of $\sim 25 \lambda$ indicates a topological charge of 25. This demonstrates that EUV vortex obtained through phase-matched absorption-limited HHG in an extended generated medium indeed retains the helical wavefront of the IR driver. Additionally, the experimental results are per perturbative conversion law $l_{q}=q l_{1}$, hence ruling out the so-called propagation effect [10] for such a hightopological charge EUV vortex even in a $15 \mathrm{~mm}$ long generation medium. 

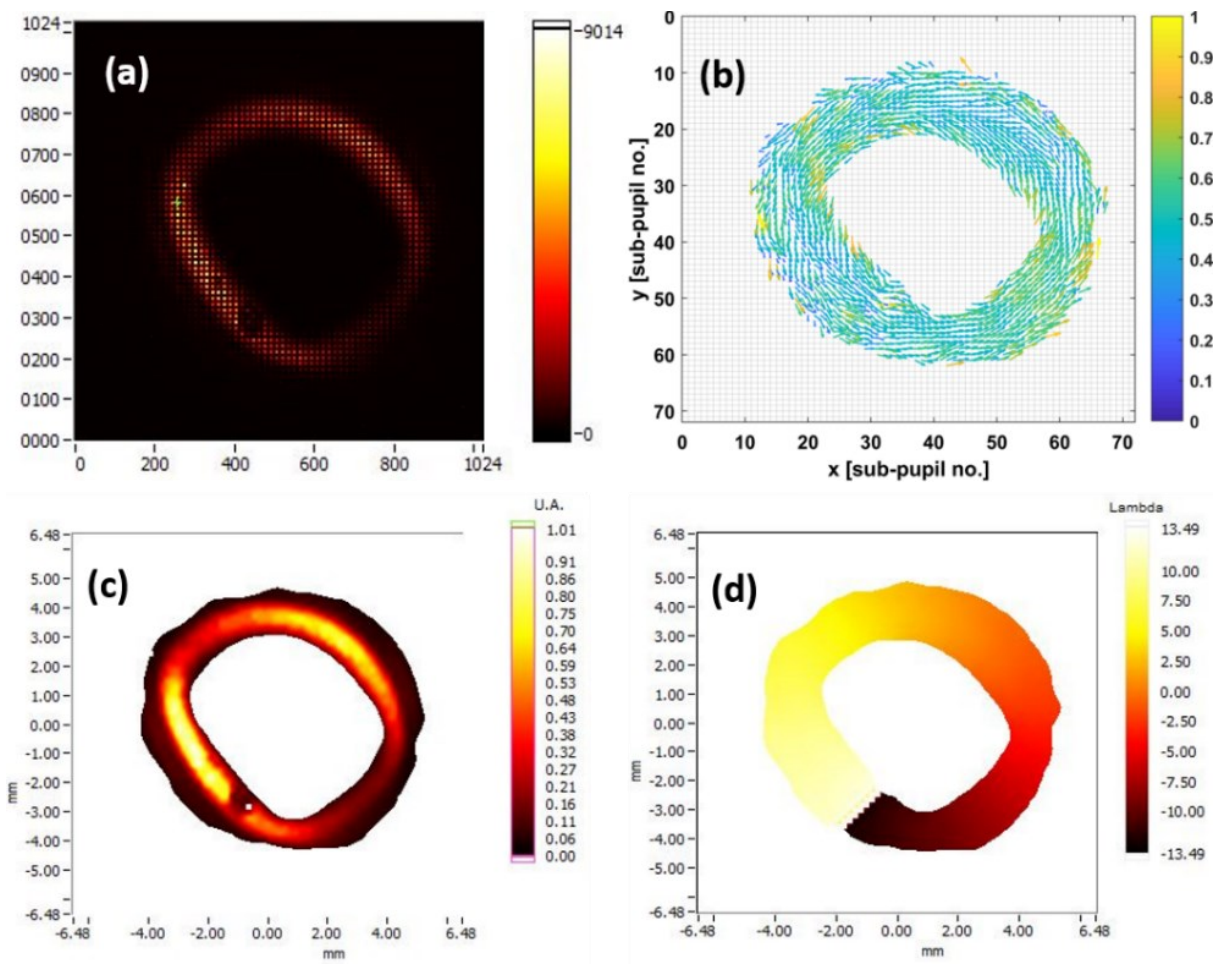

Fig. 4. HHG vortex beam generated in a $15 \mathrm{~mm}$ long Argon-filled gas-cell. Phase-matched HHG in a long generation medium permits single-shot characterization. (a) An example single-shot raw Hartmanngram of EUV vortex. (b) A quiver plot of the measured slopes indicating an anticlockwise rotation around the central singularity. (c) Reconstructed intensity profile and (d) wavefront in the unit of $\lambda$.

\section{CONCLUSIONS}

In conclusion, we have characterized intensity as well as the wavefront of high-charge EUV vortex obtained through high harmonic generation in an extended generation medium. A low numerical aperture focusing condition allowed the generation of intense vortices, hence enabling a single-shot amplitude and phase characterization. Moreover, we validate the perturbative conversion law $l_{q}=q l_{1}$ even in a $15 \mathrm{~mm}$ long generation medium, hence ruling out the possibility of any propagation effect that might affect the high-charge topological feature of the EUV vortex beam. In the forthcoming studies, single-shot characterization ability will be utilized to investigate the effect of phase-matching and other experimental parameters on the modal content of the high-charge EUV vortices.

\section{REFERENCES}

[1] Poynting, J.H., "The Wave Motion of a Revolving Shaft, and a Suggestion as to the Angular Momentum in a Beam of Circularly Polarised Light," Proc. R. Soc. Lond. A, 82, 560 (1909).

[2] Allen, L., Beijersbergen, M.W., Spreeuw, R.J.C. and Woerdman, J.P., "Orbital angular momentum of light and the transformation of Laguerre-Gaussian laser modes," Phys. Rev. A, 45, 8185 (1992).

[3] O'Neil, A. T., MacVicar, I., Allen, L. and Padgett, M. J., "Intrinsic and Extrinsic Nature of the Orbital Angular Momentum of a Light Beam", Phys. Rev. Lett. 88, 053601 (2002).

[4] Wang, X., Nie, Z., Liang, Y., Wang, J., Li, T. and Jia, B., "Recent advances on optical vortex generation," Nanophotonics, 7(9), 1533-1556 (2018).

[5] Gariepy, G., Leach, J., Kim, K. T., Hammond, T. J., Frumker, E., Boyd, R. W. and Corkum, P. B., "Creating High-Harmonic Beams with Controlled Orbital Angular Momentum," Phys. Rev. Lett., 113, 153901 (2014). 
[6] Gauthier, D., Ribic, P. R., Adhikary, G., Camper, A., Chappuis, C., Cucini, R., DiMauro, L. F., Dovillaire, G., Frassetto, F., Géneaux, R., Miotti, P., Poletto, L., Ressel, B., Spezzani, C., Stupar, M., Ruchon, T. and Ninno, G. De., "Tunable orbital angular momentum in high-harmonic generation," Nat. Commun. 8, 14971 (2017).

[7] Sanson, F., Pandey, A. K., Harms, F., Dovillaire, G., Baynard, E., Demailly, J., Guilbaud, O., Lucas, B., Neveu, O., Pittman, M., Ros, D., Richardson, M., Johnson, E., Li, W., Balcou, P. and Kazamias, S., "Hartmann wavefront sensor characterization of a high charge vortex beam in the extreme ultraviolet spectral range," Opt. Lett. 43(12), 2780-2783 (2018).

[8] Zürch, M., Kern, C., Hansinger, P., Dreischuh, A. and Spielmann, Ch., "Strong-field physics with singular light beams,". Nature Phys 8, 743-746 (2012).

[9] A. Pandey, I. Papagiannouli, F. Sanson, E. Baynard, J. Demailly, S. Kazamias, M. Pittman, O. Neveu, B. Lucas, A. Le Marec, A. Klisnick, A. Calisti, O. Larroche, D. Ros, and O. Guilbaud, "Towards subpicosecond pulses from solid target plasma based seeded soft X-ray laser," Opt. Express 28, 28924-28941 (2020).

[10]Hickmann, J. M., Fonseca, E. J. S., Soares, W. C. and Chávez-Cerda, S., "Unveiling a Truncated Optical Lattice Associated with a Triangular Aperture Using Light's Orbital Angular Momentum,” Phys. Rev. Lett. 105, 053904 (2010).

[11] Jonathan Leach, Stephen Keen, Miles J. Padgett, Christopher Saunter, and Gordon D. Love, "Direct measurement of the skew angle of the Poynting vector in a helically phased beam," Opt. Express 14, 11919-11924 (2006).

[12] Sanson, F., Pandey, A. K., Papagiannouli, I., Harms, F., Dovillaire, G., Baynard, E., Demailly, J., Guilbaud, O., Lucas, B., Neveu, O., Pittman, M., Ros, D., Richardson, M., Johnson, E., Li, W., Balcou, P. and Kazamias, S., "Highly multimodal structure of high topological charge extreme ultraviolet vortex beams," Opt. Lett. 45, 4790$4793(2020)$.

[13] Beijersbergen, M. W., Coerwinkel, R. P. C., Kristensen, M. and Woerdman, J. P., "Helical-wavefront laser beams produced with a spiral phaseplate," Opt. Commun. 112, 321-327 (1994).

[14] Rego, L., Román, J. S., Picón, A., Plaja, L. and Hernández-García, C., "Nonperturbative twist in the generation of extreme-ultraviolet vortex beams," Phys. Rev. Lett. 117(16), 163202 (2016).

[15] Meltchakov, E., Hecquet, C., Roulliay, M., Rossi, S. De., Menesguen, Y., Jérome, A., Bridou, F., Varniere, F., Ravet-Krill, M.-F. and Delmotte, F., "Development of Al-based multilayer optics for EUV," Appl. Phys. A 98, 111 (2009). 